JLAB-THY-98-48

\title{
Interpreting the Neutron's Electric Form Factor: Rest Frame Charge Distribution or Foldy Term?
}

\author{
Nathan Isgur \\ Jefferson Lab \\ 12000 Jefferson Avenue, Newport News, Virginia, 23606
}

\begin{abstract}
The neutron's electric form factor contains vital information on nucleon structure, but its interpretation within many models has been obscured by relativistic effects. I demonstrate that, to leading order in the relativistic expansion of a constituent quark model, the Foldy term cancels exactly against a contribution to the Dirac form factor $F_{1}$ to leave intact the naive interpretation of $G_{E}^{n}$ as arising from the neutron's rest frame charge distribution.
\end{abstract}




\section{INTRODUCTION}

In 1962, Sachs showed [1] that the combinations of elastic nucleon form factors $(N=p$ or $n)$

$$
\begin{gathered}
G_{E}^{N}=F_{1}^{N}-\frac{Q^{2}}{4 m_{N}^{2}} F_{2}^{N} \\
G_{M}^{N}=F_{1}^{N}+F_{2}^{N}
\end{gathered}
$$

have simple interpretations as the spatial Fourier transforms of the nucleons' charge and

magnetization distributions in the Breit frame (where momentum $\vec{p}=-\frac{\vec{Q}}{2}$ is scattered to momentum $\vec{p}^{\prime}=+\frac{\vec{Q}}{2}$ ). Here $F_{1}^{N}$ and $F_{2}^{N}$ are the Dirac and Pauli form factors, respectively, defined by

$$
\left\langle N\left(\vec{p}^{\prime}, s^{\prime}\right)\left|j_{e m}^{\mu}(0)\right| N(\vec{p}, s)\right\rangle=\bar{u}\left(\vec{p}^{\prime}, s^{\prime}\right)\left[F_{1}^{N} \gamma^{\mu}+i \frac{\sigma^{\mu \nu} q_{\nu}}{2 m_{N}} F_{2}^{N}\right] u(\vec{p}, s)
$$

where $q_{\nu}=p_{\nu}{ }^{\prime}-p_{\nu}$ and $F_{1}^{N}$ and $F_{2}^{N}$ are functions of $Q^{2}=-q^{2}$.

These form factors obviously contain vital information on the internal composition of the nucleons. Although it has proven elusive experimentally, the electric form factor of the neutron $G_{E}^{n}$ is particularly fascinating in this respect. In pion-nucleon theory, $G_{E}^{n}$ would arise from a $\pi^{-}$cloud with convection currents producing the anomalous magnetic moments $F_{2}^{p}=1.79 \equiv \mu_{p}-1$ and $F_{2}^{n}=-1.91 \equiv \mu_{n}$. In contrast, in a valence quark model, the nucleon magnetic moments arise from the underlying charged spin- $\frac{1}{2}$ constituents with the famous $S U(6)$ relation

$$
\frac{\mu_{p}}{\mu_{n}}=-\frac{3}{2}
$$

and with a scale set by

$$
\mu_{p}=\frac{m_{N}}{m_{d}} \simeq 3
$$

where $m_{d} \simeq m_{u} \simeq \frac{1}{3} m_{N}$ is a valence quark effective mass. Within this model it has been argued that in the $S U(6)$ limit $G_{E}^{n}\left(Q^{2}\right)$ would be identically zero, but that the spin-spin 
forces which produce the $S U(6)$-breaking $\Delta-N$ splitting create a charge segregation inside the neutron and lead to a nonzero $G_{E}^{n}$ [2] 团. The effect arises because the spin-spin forces push $d$ quarks to the periphery of the neutron and pull the $u$ quark to the center. Thus both the $\pi^{-}$cloud picture and the hyperfine-perturbed quark model predict a negative neutron charge radius, as observed.

Nonrelativistically, the squared charge radius is simply the charge-weighted mean square position of the constituents. More generally

$$
G_{E}^{p}\left(Q^{2}\right) \equiv 1-\frac{1}{6} r_{E p}^{2} Q^{2}+\cdots
$$

and

$$
G_{E}^{n}\left(Q^{2}\right) \equiv-\frac{1}{6} r_{E n}^{2} Q^{2}+\cdots
$$

define the proton and neutron charge radii, with

$$
\frac{G_{M}^{N}\left(Q^{2}\right)}{\mu_{N}}=1-\frac{1}{6} r_{M N}^{2} Q^{2}+\cdots
$$

defining the corresponding magnetic radii. Experimentally, the three form factors $G_{E}^{p}, G_{M}^{p}$, and $G_{M}^{n}$ are reasonably well known, although new measurements should soon determine them with much greater precision [5]. From these measurements, which cover $Q^{2}$ in the multi-GeV${ }^{2}$ range, we have learned that all three form factors have roughly similar shapes:

$$
G_{E}^{p}\left(Q^{2}\right) \simeq \frac{G_{M}^{p}\left(Q^{2}\right)}{\mu_{p}} \simeq \frac{G_{M}^{n}\left(Q^{2}\right)}{\mu_{n}} \simeq G_{D}\left(Q^{2}\right)
$$

where the dipole form factor

$$
G_{D}\left(Q^{2}\right) \equiv \frac{1}{\left[1+Q^{2} / M_{\text {dipole }}^{2}\right]^{2}}
$$

with $M_{\text {dipole }}^{2}=0.71 \mathrm{GeV}^{2}$. I note in passing that this observation makes little sense in a picture where the nucleons have a point-like core surrounded by a pion cloud (since in such a picture $G_{M}^{n}$ is purely pionic while $G_{E}^{p}$ and $G_{M}^{p}$ are mixtures of a "bare" proton and a pion cloud). In contrast, Eq. (9) is very natural in a valence quark model where, to leading order 
in a relativistic expansion, $G_{D}\left(Q^{2}\right)$ would simply be the Fourier transform of the square of the ground state spatial wavefunction.

Recent advances in experimental technique should lead to a clear measurement of the neutron's electric form factor $G_{E}^{n}\left(Q^{2}\right)$ for $Q^{2}$ in the $\mathrm{GeV}^{2}$ range in the next few years [6]. Its charge radius $r_{E n}^{2}$ is known from low energy neutron-electron elastic scattering to be $-0.113 \pm 0.005 \mathrm{fm}^{2}$ [7], but the electron-neutron scattering measurements needed to determine $G_{E}^{n}$ in the $\mathrm{GeV}^{2}$ range (in order to roughly map out the neutron's electric structure with a resolution of $10 \%$ of the proton's size) have been plagued by the lack of a free neutron target [6]. Fortunately, the method of Arnold, Carlson, and Gross [8], which uses spin observables sensitive to $G_{E}^{n}-G_{M}^{n}$ interference, opens up new methods for measuring $G_{E}^{n}$, and recent advances in accelerator, target, and detector technology are beginning to exploit these new methods.

In anticipation of these measurements, there has been renewed discussion about their interpretation. I focus here on the belief that the measured $r_{E n}^{2}$ is explained by the "Foldy term" [10. I.e., using Eqs. (1) and (7),

$$
r_{E n}^{2}=r_{1 n}^{2}+\frac{3 \mu_{n}}{2 m_{N}^{2}} \equiv r_{1 n}^{2}+r_{F o l d y, n}^{2},
$$

where $r_{1 n}^{2}$ is the "charge radius" associated with $F_{1}^{n} \simeq-\frac{1}{6} r_{1 n}^{2} Q^{2}+\cdots$. The second term in Eq. (11), called the Foldy term, appears to arise as a relativistic correction associated with the neutron's magnetic moment and so to have nothing to do with the neutron's rest frame charge distribution. It has the value $-0.126 \mathrm{fm}^{2}$, nearly coinciding with the measured value. On this basis it has been argued that any "true" charge distribution effect must be very small. In this paper I will show that while the Foldy term closely resembles $r_{E n}^{2}$ numerically, it does not "explain it". Indeed, I will demonstrate that, in the relativistic approximation to the constituent quark model in which the Foldy term first appears, it is cancelled exactly by a contribution to the Dirac form factor $F_{1}$ leaving $r_{E n}^{2}$ correctly interpreted as arising entirely from the rest frame internal charge distribution of the neutron. 


\section{THE INTERPRETATION OF THE NEUTRON CHARGE RADIUS IN A CONSTITUENT QUARK MODEL}

The relationship (1) between the Sachs form factor $G_{E}$ and the Dirac and Pauli form factors $F_{1}$ and $F_{2}$ is relativistic in origin. Unfortunately, relativistic constituent models of the nucleon are notoriously difficult: rest frame models are difficult to boost and infinitemomentum-frame (or light-cone) quark models have trouble constructing states of definite $J^{P}$. This could be the reason that the interpretation of $G_{E}^{n}$ has not been clarified in the context of such models.

While an accurate constituent quark model of nucleon structure must certainly be fully relativistic, the issue at hand can be resolved by using a relativistic expansion around the nonrelativistic limit. This is possible because the Foldy term $r_{F o l d y, n}^{2}$ arises at order $Q^{2} / m^{2}$ and so its character may be exposed by an expansion of $G_{E}^{n}$ to order $1 / \mathrm{m}^{2}$. I will also exploit symmetries of the problem available in certain limits which will make the discussion independent of the details of models.

I begin with a simple "toy model" in which a "toy neutron" $\tilde{n}_{\bar{S} D}$ is composed of a scalar antiquark $\bar{S}$ of mass $m_{S}$ and charge $-e_{D}$ and a spin- $\frac{1}{2}$ Dirac particle $D$ of mass $m_{D}$ and charge $e_{D}$ bound by flavor and momentum independent forces into a rest frame nonrelativistic $S$-wave. The calculation begins by noting that, from their definitions,

$$
G_{E}^{\tilde{n}_{\bar{S} D}}\left(Q^{2}\right)=\left\langle\tilde{n}_{\bar{S} D}\left(+\frac{Q \hat{z}}{2},+\right)\left|\rho_{e m}\right| \tilde{n}_{\bar{S} D}\left(-\frac{Q \hat{z}}{2},+\right)\right\rangle
$$

and

$$
G_{M}^{\tilde{n}_{\bar{S} D}}\left(Q^{2}\right)=\frac{m_{\tilde{n}_{\bar{S} D}}}{Q}\left\langle\tilde{n}_{\bar{S} D}\left(+\frac{Q \hat{z}}{2},+\right)\left|j_{e m}^{1+i 2}\right| \tilde{n}_{\bar{S} D}\left(-\frac{Q \hat{z}}{2},-\right)\right\rangle
$$

It is immediately clear that the calculation of these form factors requires boosting the rest frame $S$-wave bound state to momenta $\pm \frac{Q \hat{z}}{2}$. Doing so can introduce a host of $1 / m^{2}$ effects in the boosted counterpart of the $S$-wave state and it can also produce new $P$-wave-like components by Wigner-rotation of the $D$-quark spinors [9]. I will show that the latter 
effect is subleading, and will deal with the former effect by exploiting an effective chargeconjugation symmetry of the system for $m_{D}=m_{S} \equiv m$.

Since $\mu_{\tilde{n}_{\bar{S} D}}$ involves the limit of Eq. (13) as $Q \rightarrow 0$, to the required order in $1 / m$ it simply takes on its nonrelativistic value

$$
\mu_{\tilde{n}_{\bar{S} D}}=\frac{e_{D} m_{\tilde{n}_{\bar{S} D}}}{m_{D}}
$$

where of course $m_{\tilde{n}_{\bar{S} D}}=m_{S}+m_{D}$ in this limit. The Foldy term is thus well-defined:

$$
r_{F o l d y, \tilde{n}_{\bar{S} D}}^{2}=\frac{e_{D}}{2 m_{D} m_{\tilde{n}_{\bar{S} D}}}
$$

We next compute $G_{E}^{\tilde{n}_{\bar{S} D}}\left(Q^{2}\right)$ directly from Eq. (12). To leading order in $1 / m^{2}, \rho_{e m}$ remains a one-body current, and the impulse approximation is valid. Within this approximation, we make use of the relation

$$
\left\langle D\left(\vec{p}+Q \hat{z}, s^{\prime}\right)\left|\rho_{e m}\right| D(\vec{p}, s)\right\rangle=e_{D}\left(1-\frac{Q^{2}}{8 m_{D}^{2}}\right)\left\langle\tilde{D}(\vec{p}+Q \hat{z})\left|\rho_{e m}\right| \tilde{D}(\vec{p})\right\rangle \delta_{s s^{\prime}}+\rho_{\text {spin-flip }}
$$

where

$$
\rho_{\text {spin-flip }} \equiv \frac{e_{D} Q}{4 m_{D}^{2}}\left(p_{-} \delta_{s^{\prime}+} \delta_{s-}-p_{+} \delta_{s^{\prime}-} \delta_{s+}\right)
$$

and where $\tilde{D}$ is a fictitious scalar quark with the mass and charge of $D$. This expression is easily obtained by making a nonrelativistic expansion of both the $D$ and $\tilde{D}$ charge density matrix elements.

The spin-flip term $\rho_{\text {spin-flip }}$ can only contribute via transitions to and from the Wignerrotated components of the wavefunction. However, the amplitudes of such components are proportional to $Q k / m_{D}^{2}$, where $k$ is an internal momentum. Since $\rho_{\text {spin-flip }}$ already carries a factor $1 / m_{D}^{2}$, such effects may be discarded. Note that non-flip Wigner-rotated contributions are of the same order and may also be neglected.

We conclude that $r_{E \tilde{n}_{\bar{S} D}}^{2}$ may be computed by replacing $D$ by $\tilde{D}$ provided the additional contribution $-e_{D} Q^{2} / 8 m_{D}^{2}$ is added to $G_{E}^{\tilde{n}_{\bar{S} D}}$. (More precisely, this factor multiplies the $\tilde{D}$ contribution to $G_{E}^{\tilde{n}_{\bar{S} D}}$, but at $Q^{2}=0$ this is just unity.) I will denote the associated 
"zwitterbewegung" charge radius $3 e_{D} / 4 m_{D}^{2}$ by $r_{D, z w i t t e r}^{2}$. The effect of $r_{D, z w i t t e r}^{2}$ is wellknown in a variety of contexts, including atomic physics [11], nuclear physics [12], hadronic physics [13], and heavy quark physics [14]; at the most elementary and concrete level it appears as the additional factor of $\left(1-\frac{Q^{2}}{8 m_{D}^{2}}\right)^{2}$ in the ratio of the Mott cross section to the Rutherford cross section. The problem of computing the remaining contributions to $G_{E}^{\tilde{n}_{\bar{S} D}}$ to this order from the fictitious $\bar{S} \tilde{D}$ scalar-scalar bound state would in general be highly nontrivial. However, in the limit $m_{D}=m_{S} \equiv m$ these contributions vanish, since this system has in this limit a pseudo-charge-conjugation invariance under $(\bar{S}, \tilde{D}) \rightarrow(S, \overline{\tilde{D}})$. However, we note that in this limit $m_{\tilde{n}_{\bar{S} D}}=2 m$ and so from Eq. (15) we have

$$
r_{E \tilde{n}_{\bar{S} D}}^{2}=r_{D, z w i t t e r}^{2}=\frac{3 e_{D}}{4 m^{2}}=r_{F o l d y, \tilde{n}_{\bar{S} D}}^{2}
$$

i.e., in this model the "scalar charge distribution" is zero and the Foldy term would indeed account for the full charge radius of $\tilde{n}$. This conclusion is simply interpreted: the two scalar particles $\bar{S}$ and $\tilde{D}$ have perfectly overlapping and cancelling charge distributions, but the expansion of the $\tilde{D}$ distribution by $r_{D, z w i t t e r}^{2}$ creates a slight excess of $\tilde{D}$ at large radii. In terms of its experimental significance, we have concluded that in an $\bar{S} D$ model of the neutron, the observation of an equality of $r_{E n}^{2}$ and $r_{F o l d y, n}^{2}$ would indeed indicate the absence of an intrinsic "scalar" charge distribution.

We shall soon be drawing quite another conclusion for the situation in the constituent quark model. However, before leaving the $\bar{S} D$ model, it is useful to consider another limit: the "hydrogenic limit" where $m_{S} \rightarrow \infty$. In this case (see Eq. (15)), $r_{F o l d y, \tilde{n}_{\bar{S} D}}^{2}=0$ but

$$
r_{E, \tilde{n}_{\bar{S} D}}^{2}=\frac{3 e_{D}}{4 m_{D}^{2}}+e_{D} r_{w f}^{2}
$$

where $r_{w f}^{2}$ is the charge radius associated with the bound state $\tilde{D}$ problem. It may be that $r_{w f}^{2}$ contains other $1 / m_{D}^{2}$ effects, but we note that the physics of the $r_{D, z w i t t e r}^{2}$ effect previously associated with $F_{2}$ via $r_{F o l d y, \tilde{n}_{\bar{S} D}}^{2}$ now must be asociated with $F_{1}$ via $r_{1 \tilde{n}_{\bar{S} D}}^{2}$. The $\bar{S} D$ toy model thus simultaneously supplies us with a simple interpretation of the Foldy term and a warning about associating $F_{1}$ with the neutron's "intrinsic charge distribution". 
While the $\bar{S} D$ toy model has some of the characteristics of diquark models for the nucleon ( $\bar{S}$ has the quantum numbers and color of a scalar diquark), our main use for it was to introduce the basic elements of our discussion in a simple context. Indeed, it is now relatively trivial to extend our considerations to the realistic case of the valence quark model in which the neutron is in the leading approximation made of three mass $m_{q}$ spin- $\frac{1}{2}$ quarks $d d u$ bound by flavor and momentum independent forces into flavor-independent nonrelativistic relative $S$-waves. In this case

$$
\mu_{\tilde{n}_{d d u}}=-\frac{2 m_{\tilde{n}_{d d u}}}{3 m_{q}} \simeq-2
$$

so that

$$
r_{F o l d y, \tilde{n}_{d d u}}^{2}=-\frac{1}{m_{q} m_{\tilde{n}_{d d u}}}
$$

In calculating $r_{E \tilde{n}_{d d u}}^{2}$ via Eq. (12), the transformation of the calculation of the charge radius of $d d u$ to that of three scalar quarks $\tilde{d} \tilde{d} \tilde{u}$ and residual $r_{q, z w i t t e r}^{2}=-e_{q} Q^{2} / 8 m_{q}^{2}$ terms proceeds as before, as does the neglect of Wigner-rotated components of the boosted state vectors. However, in this case, since the $r_{q, z w i t t e r}^{2}$ terms are spin and flavor independent, and since the sum of the three charges is zero, they lead to no net $Q^{2} / m^{2}$ term! The reason for this is clear: the exactly overlapping and cancelling quark distributions remain exactly overlapping and cancelling after they are all equally smeared by $r_{q, z w i t t e r}^{2}$ [15. This picture also anticipates the next stage of the argument: the analog of the pseudo-charge-conjugation invariance that we used for the scalar part of the $\bar{S} D$ matrix element is that the three quark wavefunction belongs to the symmetric representation of the permutation group $S_{3}$ so that the scalar part $r_{E, \tilde{n} \tilde{d} \tilde{d} \tilde{u}}^{2}$ of the charge radius vanishes. Thus in the usual valence quark model

$$
r_{E, \tilde{n}_{d d u}}^{2}=r_{E, \tilde{n}_{\tilde{d} \tilde{d} \tilde{u}}^{2}}^{2}+\Sigma_{i} e_{i} r_{i, z w i t t e r}^{2}=0
$$

which requires that

$$
r_{1 \tilde{n}_{d d u}}^{2}=-r_{F o l d y, \tilde{n}_{d d u}}^{2}
$$


so it is appropriate to interpret the observed $r_{E n}^{2}$ as due to an intrinsic internal charge distribution. Stated in another way, the coincidence of the predicted rest frame charge distribution in such models with the experimental value of $r_{E n}^{2}$ may be claimed as a success, while the numerical coincidence of $r_{E n}^{2}$ with the Foldy term may consistently be viewed as a potentially misleading accident. Such an accident is possible because while in the nonrelativistic limit $r_{F o l d y, \tilde{n}}^{2}<<r_{E \tilde{n}}^{2}$, in QCD both constituent masses and hadronic radii are determined by $\Lambda_{Q C D}$ so they are expected to be of comparable magnitude.

\section{CONCLUSIONS AND FINAL REMARKS}

The principal conclusion of this paper is that, within the context of the valence quark model, the apparent contribution $3 \mu_{n} / 2 m_{N}^{2}$ of the Foldy term to the charge radius of the neutron is illusory: in the leading approximation it is exactly cancelled by a "nonintuitive" contribution to the radius $r_{1 n}^{2}$ of the Dirac form factor $F_{1}^{n}$. It is therefore totally appropriate to compare the measured $r_{E n}^{2}$ and in general $G_{E}^{n}\left(Q^{2}\right)$ against quark model predictions (see, e.g., Fig. 1 of the second of Refs. [3]) for the rest frame internal charge distribution of the neutron.

Before too much is made of this successful prediction of the valence quark model, some other very fundamental questions must still be answered. Perhaps the most fundamental is the possible effect of nonvalence components in the neutron wavefunction. After all, the classic explanation [16] for $r_{E n}^{2}$ is that the neutron has a $p \pi^{-}$component in its wavefunction [17] (for a discussion in the more modern context of heavy baryon chiral perturbation theory, see Ref. [18]). Since both hyperfine interactions and $q \bar{q}$ pairs are $1 / N_{c}$ effects, I know of no simple argument for why one should dominate.

Fortunately, there is both theoretical and experimental progress in resolving this old question. Recent theoretical work on "unquenching the quark model" [19 indicates that there are strong cancellations between the hadronic components of the $q \bar{q}$ sea which tend to make it transparent to photons. These studies provide a natural way of understanding the 
successes of the valence quark model even though the $q \bar{q}$ sea is very strong, and in particular suggest that the precision of the OZI rule is the result of both a factor of $1 / N_{c}$ and strong cancellations within this $1 / N_{c^{-}}$-suppressed meson cloud. New data [20] on the contributions of $s \bar{s}$ pairs to the charge and magnetization distribution of the nucleons is also beginning to constrain the importance of such effects and, by broken $S U(3)$, their $u \bar{u}$ and $d \bar{d}$ counterparts, and future experiments will either see $s \bar{s}$ effects or very tightly limit them (at the level of contributions of a few percent to $r_{E}^{2}$ and $\mu_{N}$ ). The resolution of the old question of the origin of $\mu_{n}$ and $r_{E n}^{2}$ is thus within sight.

\section{REFERENCES}

[1] R.G. Sachs, Phys. Rev. 126, 2256 (1962).

[2] R. Carlitz, S.D. Ellis, and R. Savit, Phys. Lett. 64B, 85 (1976); Nathan Isgur, Acta Phys. Pol. B8, 1081 (1977).

[3] Nathan Isgur, Gabriel Karl, and Roman Koniuk, Phys. Rev. Lett. 41, 1269 (1978); 45, 1738(E) (1980); Nathan Isgur, Gabriel Karl, and D.W.L. Sprung, Phys. Rev. D23, 163 (1981).

[4] For some variations on the theme of Refs. [2,3], see e.g., A. Le Yaouanc, L. Oliver, O. Péne, and J.C. Raynal, Phys. Rev. D11, 680 (1975); D12, 2137 (1975); D13, 1519(E) (1976); D15, 844 (1976); D18, 1733 (1978); A. Buchmann, E. Hernandez, and K. Yazaki, Phys. Lett. B269, 35 (1991); Nucl. Phys. A569, 661 (1994). For relativistic quark model variations see, e.g., H.J. Weber, Phys. Rev. D49, 3160 (1994); Z. Dziembowski, Phys. Rev. D37, 778 (1988); W. Konen and H.J. Weber, Phys. Rev. D41, 2201 (1990); F. Cardarelli, E. Pace, G. Salmè, and S. Simula, Phys. Lett. B357, 267 (1995); and for the results of a relativized quark model, see M. Warns, H. Schröder, W. Pfeil, and H. Rollnik, Z. Phys. C45, 627 (1990).

[5] See, for example, G. Quemener, C. F. Perdrisat, M. K. Jones, V. Punjabi, and the Hall A 
Collaboration at Jefferson Lab, to be published in the proceedings of the International Nuclear Physics Conference, Paris (1998); and V. Punjabi et al., BAPS 43, 1592 (1998). Preliminary results from this experiment indicate that $G_{E}^{p}\left(Q^{2}\right) / G_{M}^{p}\left(Q^{2}\right)$ drops appreciably below unity in the $\mathrm{GeV}^{2}$ region.

[6] For a recent review see F. Klein, "Recent Experiments on the Electric Form Factor of the Neutron", in Proceedings of the $14^{\text {th }}$ International Conference on Particles and Nuclei, ed. C. Carlson and J. Domingo (World Scientific, Singapore, 1997), p. 121.

[7] S. Kopecki et al., Phys. Rev. Lett. 74, 2427 (1995).

[8] R.G. Arnold, C. Carlson, and F. Gross, Phys. Rev. C23, 363 (1981).

[9] Wigner rotation is the name given to the feature that when a free Dirac particle of mass $m_{D}$ with transverse momentum $k_{T}$ is boosted by velocity $v$ along $\hat{z}$, its spin flips with an amplitude proportional to $v k_{T} / m_{D}$; see H.J. Melosh, Phys. Rev. D9, 1095 (1974) for the introduction of such rotations to the quark model.

[10] L. Foldy, Phys. Rev. 83, 688 (1951);87, 688 (1952);87, 693 (1952); Rev. Mod. Phys. 30, 471 (1958). For an example of the impact of Foldy's paper, see, e.g., the discussion on page 439 of the textbook by S. Gasiorowicz, "Elementary Particle Physics" (John Wiley, New York, 1966); for a more recent example of a discussion of the Foldy term, see F. Klein in Ref. [6].

[11] See, e.g., James D. Bjorken and Sidney D. Drell, "Relativistic Quantum Mechanics" (McGraw Hill, New York, 1964), p. 52, from which $\delta r^{2}=3 / 4 m_{e}^{2}$ follows; for a glimpse of the early literature, see T.D. Newton and E.P. Wigner, Rev. Mod. Phys. 21, 400 (1949).

[12] See, e.g., F. Gross in "Modern Topics in Electron Scattering" edited by B. Frois and I. Sick (World Scientific, New Jersey, 1991), p. 219 for the appearance of $r_{N, z w i t t e r}^{2}$ in the context of relativistic nuclear physics.

[13] See, e.g., N. Isgur and C. Hayne, Phys. Rev. D25, 1944 (1982) for the appearance of $r_{q, z w i t t e r}^{2}$ in the context of the relativized quark model. 
[14] See, e.g., J.D. Bjorken in Results and Perspectives in Particle Physics, Proceedings of the $4^{\text {th }}$ Rencontre de Physique de la Valle d'Aoste, La Thuile, Italy, 1990, edited by M. Greco, (Editions Frontieres, Gif-sur-Yvette, France, 1990) and N. Isgur and M.B. Wise, Phys. Rev. D43, 819 (1991) for the appearance of $r_{Q, z w i t t e r}^{2}$ in the context of heavy quark symmetry, where it corresponds to the fixed minimum slope of $\frac{1}{4}$ for the Isgur-Wise function.

[15] For the proton $\sum_{i} e_{i} r_{i}^{2}=3 / 4 m_{q}^{2}$, which is much larger than the Foldy term and, by contributing $\simeq 0.27 \mathrm{fm}^{2}$ to $r_{E p}^{2}$, brings the constituent quark model prediction for this quantity into better agreement with experiment [13].

[16] In its early manifestations, the pion cloud model was posited to give rise to the nucleon form factors by pion vertex corrections analogous to the QED diagrams responsible for $g-2$. See, e.g., S.D. Drell and F. Zachariasen, "Electromagnetic Structure of Nucleons" (Oxford University Press, New York, 1961); R. Hofstadter, "Nucleon and Nuclear Structure" (W.A. Benjamin, Inc., 1963); L. Hand, D.G. Miller, and R. Wilson, Rev. Mod. Phys. 35, 335 (1963); S.D. Drell, in Int. School of Physics "Enrico Fermi", Course XXVI, Varenna, 1962 (Academic Press, New York, 1964). As explained in this work, the strong $\pi \pi$ interactions in the $t$-channel call for dispersion-theoretic approach. See G.F. Chew, R. Karplus, S. Gasiorowicz, and F. Zachariasen, Phys. Rev. 110, 265 (1958), and P. Federbush, M.L. Goldberger, and S.B. Treiman, Phys. Rev. 112, 642 (1958). These papers in turn led to the development of the vector meson dominance model. For a sense of the degree of dominance of the meson cloud picture of the nucleon form factors before the discovery of the quarks, see the figure on page 451 of the undergraduate textbook "Mechanics", Berkeley Physics Course - Volume 1, by Charles Kittel, Walter D. Knight, and Marvin A. Ruderman (McGraw Hill, New York, 1965): the point-like proton core and pion cloud of both the proton and neutron are evident and stated to be based on high-energy electron scattering data.

[17] I have examined the Foldy term in a model of a nonrelativistic $P D L=1$ bound state, where $P$ is a pseudoscalar particle. In such a system (which represents the most naive version of a 
$p \pi^{-}$model for the neutron), $\rho_{\text {spin-flip }}$ makes a contribution which exactly cancels $r_{D, z w i t t e r}^{2}$ ! Thus in the pseudo-charge-conjugation symmetry limit $m_{P}=m_{D} \equiv m, r_{E \tilde{n}_{P D}}^{2}=0$ and, as in the quark model, one has $r_{1 \tilde{n}_{P D}}^{2}=-r_{F o l d y, \tilde{n}_{P D}}^{2}$ so that any observed $r_{E n}^{2}$ may be interpreted as arising from an intrinsic internal charge distribution (as naively pictured in the $p \pi^{-}$model).

[18] See, e.g., V. Bernard, H.W. Fearing, T.R. Hemmert, and U.-G. Meissner, Nucl. Phys. A635, 121 (1998) and references therein.

[19] P. Geiger and N. Isgur, Phys. Rev. D 41, 1595 (1990); P. Geiger and N. Isgur, Phys. Rev. D 44, 799 (1991); Phys. Rev. Lett. 67, 1066 (1991); Phys. Rev. D 47, 5050 (1993); P. Geiger, ibid. 49, 6003 (1993); P. Geiger and N. Isgur, Phys. Rev. D55, 299 (1997).

[20] "Measurement of the Neutral Weak Form Factors of the Proton", The HAPPEX Collaboration, K.A. Aniol et al., submitted to Phys. Rev. Lett. 